\title{
REVIEW
}

Open Access

\section{The effects of opium on the cardiovascular system: a review of side effects, uses, and potential mechanisms}

\author{
Samaneh Nakhaee ${ }^{1}$, Saeedeh Ghasemi ${ }^{2}$, Kimiya Karimzadeh², Nasim Zamani ${ }^{3}$, Samaneh Alinejad-Mofrad ${ }^{4}$ and \\ Omid Mehrpour ${ }^{1,5^{*}}$
}

\begin{abstract}
Background: In Iran, as in many other Asian and Middle Eastern countries, some believe that opium has beneficial effects on cardiovascular system. Dependent patients suppose that opium has positive effects on cardiovascular function and can prevent or improve cardiovascular diseases; however, only few comprehensive studies evaluating such effects have been performed.
\end{abstract}

Objectives: In this study, we sought to clarify the effect of opium on cardiovascular problems by incorporating the previous findings and the current information on the issue and to explain the possible mechanisms of this effect.

Methods: The available human studies published up to October 30, 2019, were searched in different databases. Case-control, cohort, and cross-sectional studies were retrieved. Papers published in English or those with an English abstract were included. The risk of bias for each included study was assessed based on the NewcastleOttawa Scale (NOS). We then categorized the effects of opium on cardiovascular problems along with its probable underlying mechanisms of action.

Results: In this study, most of the published articles suggested the adverse effects of opium on the cardiovascular system, including atherosclerosis, myocardial infarction, arrhythmia, low ejection fraction, and cardiovascular mortality; however, some articles reported the beneficial or impartial effects of opium on the cardiovascular system. In this article, we have categorized all the effects of opium on cardiovascular system; also, the proposed mechanisms of action of opium in each of the above-mentioned disorders are summarized.

Conclusion: Although the available evidences were incoherent, it was mostly suggested that opium use does not protect against or improve cardiovascular problems.

Keywords: Opium, Cardiovascular diseases, Coronary artery disease

\footnotetext{
* Correspondence: omid.mehrpour@yahoo.com.au

${ }^{1}$ Medical Toxicology and Drug Abuse Research Center (MTDRC), Birjand

University of Medical Sciences, Birjand, Iran

${ }^{5}$ Rocky Mountain Poison and Drug Safety, Denver Health and Hospital

Authority, 1391 Speer Blvd, 777 Bannock St. MC 0180, Denver, CO 80204,

USA

Full list of author information is available at the end of the article
}

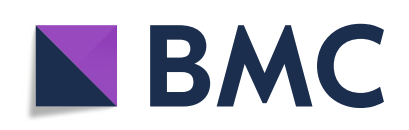

(0) The Author(s). 2020 Open Access This article is licensed under a Creative Commons Attribution 4.0 International License, which permits use, sharing, adaptation, distribution and reproduction in any medium or format, as long as you give appropriate credit to the original author(s) and the source, provide a link to the Creative Commons licence, and indicate if changes were made. The images or other third party material in this article are included in the article's Creative Commons licence, unless indicated otherwise in a credit line to the material. If material is not included in the article's Creative Commons licence and your intended use is not permitted by statutory regulation or exceeds the permitted use, you will need to obtain permission directly from the copyright holder. To view a copy of this licence, visit http://creativecommons.org/licenses/by/4.0/. The Creative Commons Public Domain Dedication waiver (http://creativecommons.org/publicdomain/zero/1.0/) applies to the data made available in this article, unless otherwise stated in a credit line to the data. 


\section{Background}

Opium is a crude material retrieved from Papaver somniferum, which is quite effective in the treatment of acute and chronic pain but can also result in opium use disorder [1]. Opium use is a social and health problem in many countries, including Iran [2, 3], and it has been reported to be the most commonly used substance in this country $[4,5]$. Opium mostly affects the central nervous system (CNS), but it can also affect the respiratory and cardiovascular systems [6-8].

In many Asian and Middle Eastern countries, including Iran, some believe that opium has beneficial effects on cardiovascular diseases (CVDs) although only few studies have been performed to confirm or reject this assumption [1]. Studies show that patients with chronic diseases, including diabetes, hypertension, and ischemic heart disease (IHD), use opium more than the general population because they assume it is efficient in treating or preventing cardiovascular disorders. This is even a common belief among the medical community [9].

Therefore, we have tackled an increasing trend toward opium use in our society and the results obtained from the very limited studies on the issue are contradictory. The aim of this review article was, therefore, to clarify the effect of opium on cardiovascular problems by integrating the previous findings and the current information on the issue and to explain the possible mechanisms of this effect.

\section{Methods}

\section{Search strategy}

In this systematic review performed in accordance with the instructions of Preferred Reporting Items for Systematic Reviews and Meta-Analyses (PRISMA), several databases including PubMed, Web of Science, Scopus, Embase, Ovid, Google Scholar, and Persian databases such as Magiran and SID were searched. We used $\mathrm{MeSH}$ headings, free-text terms, and combination of relevant keywords including "opium", "papaver", "opiate”, “opioid", "coronary heart disease", "coronary artery disease", "myocardial infarction", "cardiovascular", "atherosclerosis", "atherogenesis", and "ischemic heart disease". PICO frame of this review was defined as P: Opium user, C: Non-opium user, O: Cardiovascular Diseases risk.

For example, the search strategy in PubMed central using MeSH terms and free-text terms were as follow:

(((“Opium”[MeSH]) OR “papaver”[MeSH])) AND ( ((()((“cardiovascular diseases”[MeSH] $)$ OR “Coronary Artery Diseases"[Mesh]) OR "myocardial infarction”[Mesh]) OR "Atherosclerosis"[MeSH]) OR "Myocardial Ischemia”[$\mathrm{MeSH}])$ OR "coronary artery bypass surgery" [MeSH]) OR "percutaneous coronary intervention" $[\mathrm{MeSH}])$

(((()((“cardiovascular disease”[Title/Abstract]) OR “Coronary Artery Disease"[Title/Abstract]) OR "myocardial
infarction"[Title/Abstract]) OR “Atherosclerosis"[Title/ Abstract]) OR "percutaneous coronary intervention"[Title/Abstract]) OR “Myocardial Ischemia”[Title/Abstract]) OR "coronary artery bypass surgery"[Title/Abstract])) AND (((opium [Title/Abstract]) OR papaver [Title/Abstract]) OR opioid [Title/Abstract]). Two researchers independently searched the above-mentioned databases until October 30, 2019.

\section{Inclusion criteria and study selection}

Case-control, cohort, and cross-sectional studies were retrieved. In order to find further articles on the subject, reference lists of the identified articles were also scanned. No restrictions were imposed on the language and study period. Papers published in English or those with an English abstract were included. Studies about natural opioids such as morphine and synthetic opioids such as methadone were not included in our review. Also, articles with inadequate data and no clear description of the methods, editorials, conference papers, and review articles were excluded. Irrelevant studies were excluded from the list by the assessment of their titles, abstracts, and full texts. The screening was performed after exclusion of duplicates. The primary outcome was defined as the risk of atherosclerosis, myocardial infarction, arrhythmia, effects on the ejection fraction index, the severity of $C A D$, and mortality from CADs (Table 1 ). We included studies assessing at least one of these primary outcome measures. The flowchart of our searching process for the selection of the included articles is presented in Fig. 1.

\section{Data extraction}

Data were extracted using a pre-defined checklist and were assessed by two independent reviewers. The data consisted of the date and place of the study, type of study, sample size and sampling method, information about the participants, the definition of exposure and outcome, route and amount of exposure, age, and gender of the participants, methods of assessment, confounding variables, and the main findings of each study with principal measures (e.g., risk ratio, difference in means, etc.). The risk of bias for each included study was assessed based on the Newcastle-Ottawa Scale (NOS). Studies with a score of 5 or more were considered as high-quality. None of the articles meeting our inclusion criteria were excluded. Finally, we tried to categorize the effects of opium on cardiovascular problems along with identifying its probable underlying mechanisms of action (Fig. 2).

\section{Results}

Opium and atherosclerosis

Some previous studies mention that opium is a risk factor for atherosclerosis, while others advocate its positive 
Table 1 Opium and Cardiovascular problems

\begin{tabular}{lll}
\hline Opium and & Potential risk factor & {$[10-18]$} \\
& Potential protective factor & {$[1],[19]$} \\
& Impartial factor & {$[20-25]$} \\
MI & Potential risk factor & {$[26-31]$} \\
& Potential protective factor & {$[32-35]$} \\
& Impartial factor & {$[36-40]$} \\
& Potential risk factor & {$[41],[37],[21],[39],[42],[43],[44],[45]$} \\
Arrhythmias & Potential protective factor & {$[46]$} \\
& Impartial factor & {$[47]$} \\
& Potential risk factor & {$[24],[22],[21,48],[42],[49],[50]$} \\
Ejection Fraction & Impartial factor & {$[1],[12],[26],[32,47],[16],[3,51],[38],[33],[23,39,52-54]$} \\
& Potential risk factor & {$[21],[24],[37],[39],[55]$}
\end{tabular}

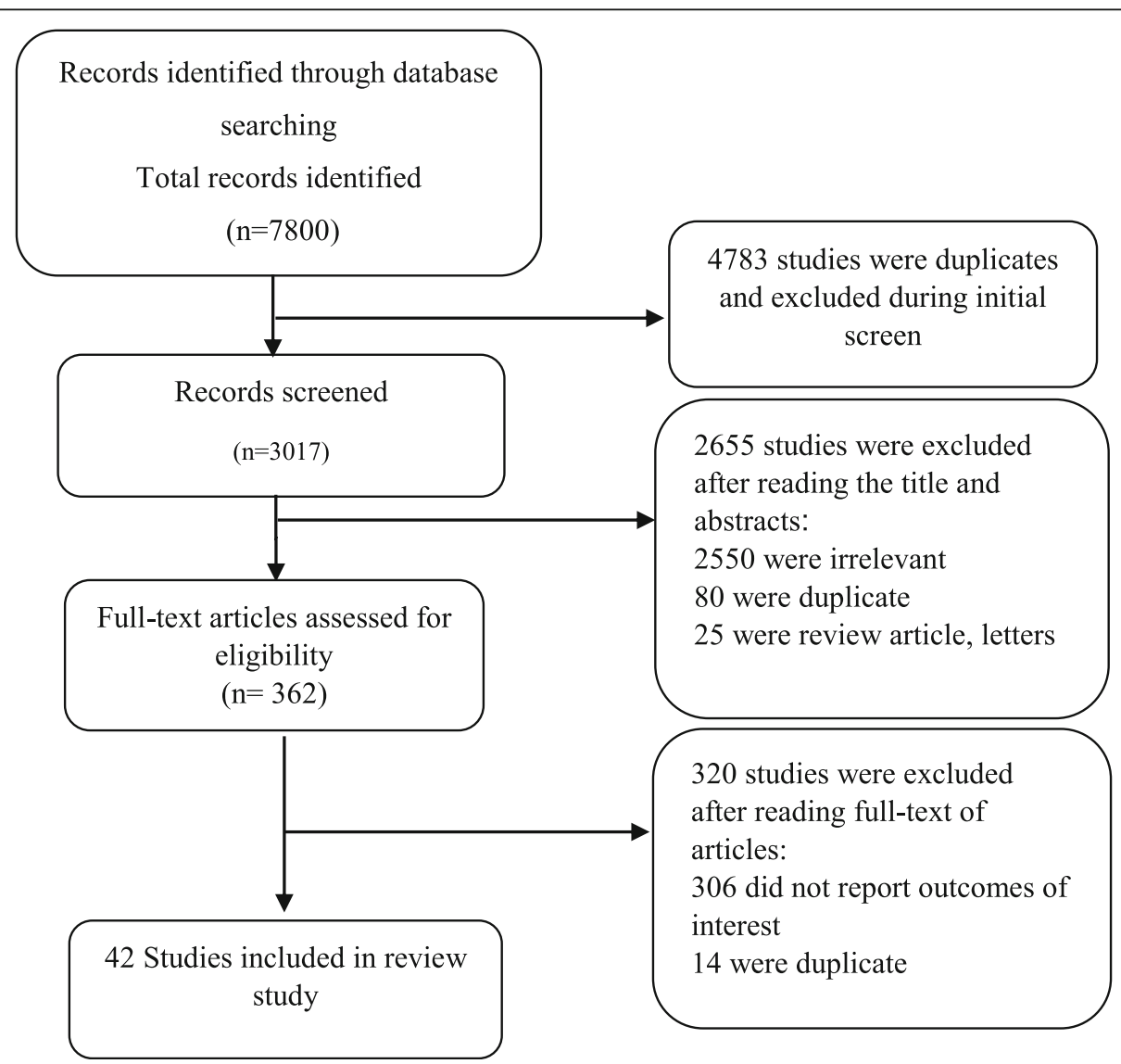

Fig. 1 Flowchart of the literature search and strategy for the selection of the relevant documents 


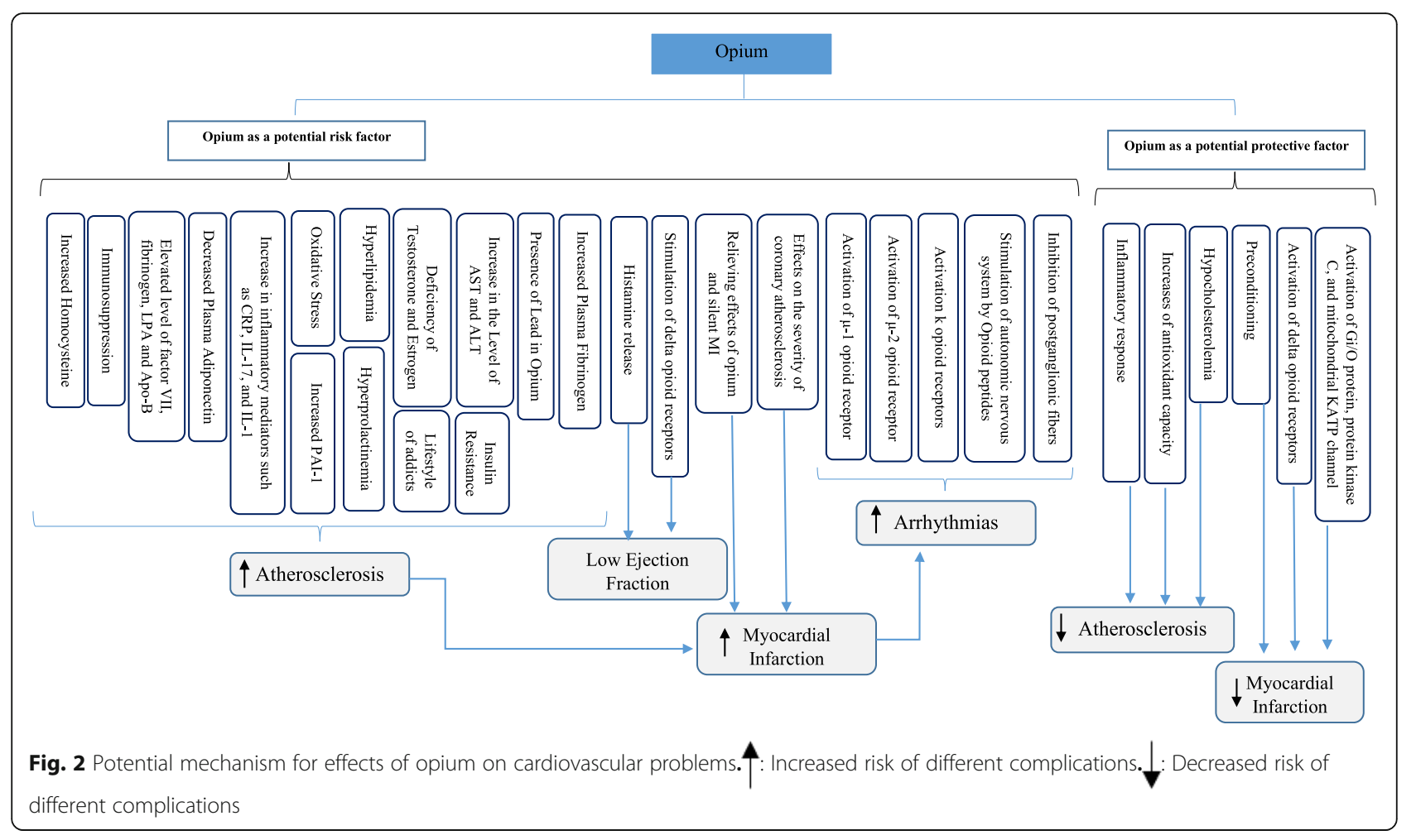

effects. There are also a few studies ascribing that opium does not affect atherosclerosis.

\section{Opium as a potential risk factor for atherosclerosis}

A study of 2405 patients showed that using opium was a risk factor for CADs (OR:1.8, CI: 1.1-3.1, $P=0.015$ ). This study also excluded cigarette smokers from the analysis and obtained the same general findings. The amount of the consumed opium was found to be significantly associated with the severity of atherosclerosis $(\mathrm{r}=$ $0.2, P=0.002)[10]$. In another study, the risk factors for CADs were evaluated in young men and women undergoing angiography. Patients were categorized based on CADs, and the results showed that CADs were related to diabetes and increased LDL and lipoprotein $\mathrm{A}$ in women and cigarette smoking and opium use in men. In all the patients, opium use was the second significant factor affecting CADs following diabetes (OR:2.65, CI: $1.31-5.35, P=0.007)$ [11].

The results of a cross-sectional study of 1925 diabetic patients undergoing angiography (228 opium users and 1697 non-users) showed that CADs were more severe in opium dependent patients. The average Gensini score and the extent score used to assess the severity of CADs were significantly higher in those with opium use disorder. There was no relationship between the route of opium use (inhaled or orally consumed) and the severity of CADs, but the consumed dose of opium was related to this variable [12].
Darabad et al. investigated 1170 patients undergoing coronary artery angiography (121 and 1049 with and without a history of opium use, respectively). The researchers concluded that older age (OR:0.68,P:0.00), diabetes (OR:2.416, P:0.00), hypertension (OR:2.007, P: 0.000) and opium use (OR:2.083, P:0.019) were the risk factors for CADs although no difference was detected in the number of the involved coronary arteries [13]. In another study, 242 patients undergoing angiography were investigated. The relative frequency of stenosis $\geq 50 \%$ and the number of the involved arteries were significantly higher in dependent patients. Multiple logistic models of age, gender, and opium use demonstrated a significant relationship between opium use and CADs [14].

Nadimi et al. evaluated 250 patients with evidence of stable angina, heart ischemia in a cardiac stress test, and class I or II symptoms in coronary angiography. The patients were examined in two 125-patient groups; one with microvascular coronary dysfunction (MCD) and the other with normal angiography and no MCD. The two groups were homogeneous regarding gender, age, history of diabetes, blood pressure, and blood lipids. Based on the results, opium use disorder was reported to be a prognostic factor for MCD (OR $=3.575$, CI 95\%: $1.161-$ 9.016, $P=0.0069$ ) [15].

Another study was performed to determine the association between acute opium use and CADs in 91 candidates for angiography. Those with positive results in catheterization were selected as cases and those with 
normal angiography were assigned to the control group. The two groups were similar in demographic information and risk factors. The results showed that opium use disorder was a risk factor for CADs (OR: 3.8, CI 95\%: 1.5-9.5). In cases, the number of involved arteries and the degree of coagulation in them were not significantly different between dependent and non-dependent patients [16]. The results of an experimental study revealed a surge in the levels of triglycerides (TG), cholesterol, LDL, and atherogenic index of plasma in non-addicted and addicted hypercholesterolemic rabbits as time passed, with a significantly more severe deterioration in addicted hypercholesterolemic rabbits. Arteriosclerotic plaques of the aorta were significantly more in the addicted hypercholesterolemic rabbits.. It was concluded that oral consumption of opium had aggravating effects on the formation of arteriosclerotic plaques and exacerbated the atherogenic effects of high-fat foods [17]. Another study evaluating postmortem heart diseases in 168 addicted bodies showed that of the 35 cases with CADs, 28 had significant stenosis in one to more than four coronary arteries due to arteriosclerotic plaques [18].

\section{Potential mechanisms}

Inflammation Atherosclerosis depends on the immune response [59]. Some studies on substance use disorder consider opium as an immunosuppressive factor [59] and reveal an increase in inflammatory mediators such as C-reactive protein (CRP), interleukin-17, and interleukin-1 receptor antagonist in opium users. Thus, aggravation of inflammation increases atheroma formation in dependent patients [40]. In a study of 15 dependent and 15 non-dependent patients with involved coronary arteries and an ejection fraction of more than $35 \%$, the plasma levels of interleukin- 6 and interleukin-1 receptor antagonists were evaluated. The level of interleukin-1 receptor antagonist was higher in the dependent cases, but opium did not have a significant effect on the plasma level of interleukin-6 [59]. Thirty-five dependent smokers were compared to 35 nondependent smokers in another study, which revealed that the level of factor VII was significantly higher in addicted cases [60]. One study showed that the levels of CRP, factor VII, fibrinogen, lysophosphatidic acid (LPA), and apolipoprotein $\mathrm{B}$ (Apo-B) were higher in patients with opium use disorder, while the levels of LDH and Apo-A were reported to be significantly lower in them compared to non-users [40].

Hyperlipidemia Opium consumption may have undesirable effects on lipid metabolism [61, 62]. Some clinical studies have shown that opium may affect the levels of total cholesterol, TG, LDL cholesterol, and HDL cholesterol [17, 32, 63-65].

Increase in the levels of aspartate (AST) and alanine aminotransferase (ALT) substance use may increase the levels of AST and ALT, which in turn, can cause severe atherosclerosis in the coronary arteries [3].

Oxidative stress Oxidative stress plays a critical role in the pathogenesis of atherosclerosis. It has been advocated that morphine and heroin decrease the total antioxidant capacity irrespective of cigarette smoking. Therefore, elevated oxidative stress may involve in atherosclerosis in opium users [40].

Increased Homocysteine Increased homocysteine is one of the factors that can result in the advancement of atherosclerosis and the development of CADs. It has been demonstrated that opium use disorder is strongly related to increased homocysteine, and consequently, the elevated risk of developing CADs [66].

Increased plasma fibrinogen Increased plasma fibrinogen is also considered as an independent risk factor for CADs and can increase the risk of thrombosis. Some studies have reported that plasma fibrinogen is significantly higher in opium-addicted patients [67-69].

Increased plasminogen activator Inhibitor-1 (PAI-1) PAI-1 inhibits the formation of plasmin through inhibitory effects on plasminogen activator and consequently prevents the clotting cascade in the arteries. A study conducted among 160 patients with CADs showed that the average serum level of PAI-1 was higher in addicted patients with congestive heart disease (CHD) [70].

Decreased plasma Adiponectin Adiponectin has antidiabetic and anti-atherogenic effects, and when decreased, it promotes the risk of developing metabolic disorders such as insulin resistance and cardiovascular diseases (CVDs) in patients with opium use disorder. The relationship between adipokinase and opium use disorder can indirectly play a role in the development of atherosclerosis. One case-control study demonstrated that the serum level of adiponectin was significantly lower in patients with opium use disorder [71].

Deficiency of testosterone and estrogen In men, low testosterone level can cause CADs and increase the risk of cardiovascular-related mortality. The level of plasma testosterone is lower in those with opium use disorder. Similarly, low level of estrogen and reduction of estrogen in the pre-menopause period have been reported in addicted females, which can cause CADs [40]. 
Hyperprolactinemia Opium increases the level of prolactin in the blood. Prolactin can induce smooth muscle cells growth through a mechanism that depends on protein kinase $\mathrm{C}$ and may also play a role in the hyperplasia of muscle cells of the arteries and lead to atherosclerosis [40].

Insulin resistance Similar to type 2 diabetic patients, people with opium use disorder show insulin resistance and an increase in the insulin level of the blood. Insulin resistance results in the atherosclerosis of coronary arteries and the advancement of coronary plaques [40].

Presence of Lead in opium The presence of impurities such as lead in opium can be a factor causing adverse effects on the cardiovascular system. Lead has been reported to be present in opium samples, and blood lead level has been shown to be high in those with opium use disorder [72-74]. Some studies have indicated a relationship between blood lead concentration, hypertension, and development of atherosclerosis [75].

Lifestyle Opium is a CNS suppressant and can decrease physical activity in addicted individuals, which increases the risk of CVDs [40]. In a study, depression, reduction of physical activity, and obesity were all reported in addicted patients [76]. Also, some studies showed that opium users followed treatment and nutrition advice and performed cardiovascular activities less than others $[3,51]$.

\section{Opium as a potential protective factor}

One study investigating the effects of long-term use of opioids on the extent of CADs showed that the coronary arteries clogged less severely in opioid dependents [19]. In a cross-sectional study conducted on 325 patients who were candidates for coronary artery bypass grafting ([CABG], 117 opium users and 208 non-users), fewer coronary arteries were involved in opium users, and there was no relationship between the number of involved coronary arteries and stenosis and long-term opium use [1].

\section{Potential mechanism}

Some studies reflect that opioids can reduce inflammation and CADs $[19,40]$. It has been stated that longterm opium use reduces atherosclerosis directly and protects against ischemic and infarction damages [19]. As there are more than 40 different alkaloids in opium, more research is required to better understand the effect of opium on the inflammatory response. Several other studies have shown that opium increases serum antioxidant capacity [77]. Gülçin et al. also reported a robust antioxidant capacity for morphine in vitro [78]. Several studies have demonstrated a remarkable hypocholesterolemia pattern in patients with substance use [79]. All of the issues mentioned above mandate the necessity of more in-depth research on this subject.

\section{Opium as an impartial factor}

Some studies deem the effects of opium on the development of atherosclerosis to be insignificant. A study on 299 candidates for coronary angiography showed that opium users had a higher chance of acute CADs, although this relationship was not statistically significant [20]. In a study of 268 patients with CADs, it was revealed that smoking and recent myocardial infarction (MI) were more common in patients with opium use disorder, and hyperlipidemia and diabetes were more common in patients without opium use disorder. The results showed that there was no statistically significant difference in the number of involved coronary arteries between the two groups of people with and without opium use disorder [21].

The results of another study on 1339 patients who were candidates for coronary artery bypass showed that the number of involved coronary arteries, more than $50 \%$ stenosis of the main left artery, and the extent of carotid stenosis were not different between patients with and without opium use disorder [6]. A study on 232 CAD patients showed that the number of involved coronary arteries was similar between these two groups [22]. Rezvani (2012), in a cross-sectional study on 81 patients suffering from IHD, showed that orally consumed (OR: 0.91, CI 95\%: 0.508-1.65, P: 0.88) or inhaled opium (OR:1.4, CI95\%: 0.65-3.02, P: 0.39) did not significantly affect IHD. Oral consumption of opium was a protective factor against stroke but not against IHD [23]. In a study of 566 patients undergoing CABG, it was demonstrated that patients with a positive history of opium use $(82$ people) were similar to non-users (484 people) regarding the number of the involved arteries [24].

Shahryari conducted an experimental study on male hamsters to investigate the influence of daily opium use on lipid profile and aortic atherosclerosis in one month. The results showed that the levels of LDL and TG were significantly higher, and HDL level was significantly lower in opium dependent hamsters than the control group. In addition, histopathologic changes in the heart (i.e., fatty streak, fibrous plaque, and calcification) were not different between the groups [25].

\section{Opium and MI}

Myocardial infarction occurs due to a block in the blood flow to a part of the myocardium. There are different risk factors for this condition, and some believe that endorphin and endogenous opioids play an important role 
in the development of MI, while others point to their protective or ineffective role in the development of MI.

\section{Opium as a potential risk factor}

Roohafza et al. investigated 469 patients suffering from acute MI in a cross-sectional study and concluded that opium use disorder reduced the age of MI up to 3.6 years $(P=0.003$, CI 95\%:1.2-6.0) [26]. In a case-control study, $118 \mathrm{MI}$ patients in the coronary care unit were compared with 118 heart-healthy patients admitted to other wards. The results showed that opium use had a relationship with $\mathrm{MI}(\mathrm{OR}=26.3,95 \%$ CI:7.5-92.4, $P<$ 0.01 ), but the type of MI and its extent were not statistically different between patients with and without opium use disorder in the group suffering from MI [27].

Another nested case-control study conducted among 11,693 patients suffering from MI showed that using opioids increased the risk of MI up to 1.28 times $(95 \%$ CI: 1.19-1.37) and this risk was higher in patients receiving morphine (OR: 1.71, CI 95\%: 1.09-2.68), meperidine (OR: 2.15, CI 95\%: 1.24-3.74), and mixed treatments (OR: 1.46, CI 95\%: 1.22-1.76) [28]. Bartolucci et al. performed a study on 18,048 MI patients, 285 of whom were opium users. They showed that users had a significantly higher risk of ST-elevation and infarction in the anterior wall [29]. Another study demonstrated that creatinine phosphokinase, lactate dehydrogenase, and $\mathrm{CKmb}$ enzymes were higher in patients with opium use disorder and acute MI [30].

An experimental study evaluated the effects of inhaled opium on rabbits and showed that using opium increased the level of troponin-I and exacerbated electrocardiographic (ECG) changes. It was also revealed that long-term use of opium raised the frequency of ST elevation. Exposure to opium reduced myocardium degeneration after the induction of ischemia but increased tissue congestion and hemorrhage [31].

\section{Potential mechanism}

The high prevalence of ischemic changes in ECG (ST-T changes in the ECG) and increased heart enzymes in patients with opium use disorder can be related to the relieving effects of opium on chest pain and the high prevalence of silent MI in these patients [1]. Also, the relationship between opium use and MI can be a result of opium effects on the development of coronary atherosclerosis.

\section{Opium as a potential protective factor}

A study among 460 patients suffering from acute MI (239 people with opium use disorder and 221 without opium use disorder) revealed that the emergence of MI in the anterior wall was significantly less common in opium users. The emergence of posterior wall infarction was similar between the two groups [32]. The results of a retrospective study investigating the information of 1545 men undergoing percutaneous coronary intervention (PCI) from the data bank of Tehran Heart Center showed that the ratio of involvement of the left anterior descending artery was higher in non-users. However, the reference vessel diameter and lesion length were not different between the two groups [33]. Another experimental study on the rabbit heart showed that morphine reduced cardiomyocyte apoptosis through the activation of delta-opioid receptors [34]. Research on the mouse heart showed that kappa opioid receptors in the heart caused enlargement in the infarction area, while deltaopioid receptors had protective effects [35].

\section{Potential mechanism}

Activation of delta-opioid receptors prior to ischemia reduced the infarct area in several animal models, including rats [80], rabbits [81], and pigs [82]. Recent data has also shown that opioid peptides are involved in a phenomenon called preconditioning, which protects the heart from the damages of long-term ischemia by temporary ischemia or hypoxia and reduces the size of the infarct area. Shultz et al. mentioned that the induction of preconditioning through morphine and delta- 1 opioid receptor agonists proved the role of opioid peptides in the preconditioning and cardioprotective effect [83, 84]. It has also been demonstrated that delta receptorselective agonists enforce protective effects in the heart myocytes through the activation of $\mathrm{Gi} / \mathrm{O}$ protein, protein kinase $\mathrm{C}$, and finally, the mitochondrial KATP channel [85].

\section{Opium as an impartial factor}

A case-control study conducted among $150 \mathrm{MI}$ patients hospitalized in the intensive care unit (ICU) and 150 patients hospitalized in the surgery ward showed that opium use disorder was not significantly different between the cases and controls $(\mathrm{OR}=1.71, P>0.05)$ [36]. Also, the results of another study on $116 \mathrm{MI}$ patients showed that the number of extensive infarctions was twice in people with opium use disorder, but this difference was statistically insignificant [37]. Another study on 160 males diagnosed with acute MI also confirmed that there were no differences between those with and without opium use disorder in terms of location of the MI, angiography findings, and the level of troponin-I [38]. Rostamzadeh and Khademvatani reported that there was no statistically significant difference between the two groups of opium users and non-users regarding the prevalence of ST-elevated MI (STEMI), location of MI, and the peak amount of CPK-MB [39]. Overall, the majority of the studies pinpoint opium as a risk factor for MI. This substance can hide MI symptoms through its 
pain-relieving properties, and consequently, delay treatment and cause lesion progression [40].

\section{Opium and arrhythmias Opium as a potential risk factor}

It has been previously mentioned that opium use disorder is a risk factor for the incidence of arrhythmias after acute MI irrespective of the patients' age, gender, ejection fraction, and the extent of infarction $(P=0.001$; CI: 7.26-28.65; OR =14.60). Premature ventricular contractions (PVCs) and ventricular tachycardia (VT) are significantly more common in opium-addicted individuals [41]. The results of a cross-sectional study on 116 patients suffering from MI revealed that ventricular arrhythmias such as PVCs, VT, and ventricular fibrillation (VF) were significantly more prevalent in patients with opium use disorder [37]. Another study on 268 addicted and non-addicted CAD patients showed that complications after cardiac arrhythmia surgery were significantly higher in opium users [21]. Also, it was proved that VT and VF were more common in dependent patients following MI [39]. In patients with opium and ephedrone use, sinus tachycardia, ventricular extrasystole, and supraventricular extrasystole were diagnosed in 4.67, 7.4, and $6.11 \%$ of patients, respectively [42]. Another crosssectional study showed that 60.6 and $54.6 \%$ of patients with opium and methadone toxicity had QT prolongation, respectively [86].

The combined effect of opium use and hypercholesterolemia was investigated on fatal heart arrhythmia in an experimental study on rabbits, which showed that longterm use of opium increased the atherogenic index of plasma. For a short period, QT interval increased significantly in the hypercholesterolemic group, but the index was similar in the two groups in the long run [43]. The results of another study showed that morphine and pentazocine had arrhythmogenic effects on the pig heart [44]. A case report demonstrated atrial flutter in an infant whose mother was addicted to cocaine and opiates. The infant was born to a 35-year-old mother through cesarean section (due to fetal tachycardia) in the 36th week of gestation and weighted $3.1 \mathrm{~kg}$. It had atrioventricular tachycardia, and ultimately, an atrial flutter developed [45].

\section{Potential mechanisms}

The initial pathophysiology of arrhythmia is related to dysfunction in the conduction system. Opioid receptors in the ventricles and atria may play an essential role in the development of different arrhythmias $[87,88]$ by increasing heart rate and causing changes in heart rhythm $[89,90]$. It has also been claimed that the $\mu-1$ opioid receptor causes tachycardia, and $\mu-2$ causes bradycardia [91]. Based on evidence, kappa opioid receptors can be involved in the development of an arrhythmic response. Coles et al. showed that the activation of kappa opioid receptors in pigs caused arrhythmia in them. They demonstrated that neither pentazocine nor morphine had protective effects on the heart, and the pro-arrhythmic effects of these medications were removed through blockage of the $\mathrm{k}$ receptors [44].

Several studies suggest that the potential arrhythmogenic activities of opioids at small doses occur through the activation of kappa opioid receptors, and their antiarrhythmic actions at larger doses occur due to direct interaction with heart cell membranes [92]. Opioid peptides have also been advocated to stimulate the autonomic nervous system and raise heart rate and systolic blood pressure, which may cause arrhythmia per se [87]. Endogenous opioids, together with the sympathetic and parasympathetic systems in the brain stem, control the cardiovascular function. The postganglionic fibers innervating the rabbit heart have been shown to contain opioid and cannabinoid receptors, through the inhibition of which, neurogenic tachycardia ensues [93].

\section{Opium as a potential protective factor}

In a cross-sectional study on the patients undergoing CABG, it was revealed that using opium was one of the factors that significantly prognosticated atrial fibrillation after CABG (OR = 0.37, 95\% CI: 0.93-1; $P=0.02)$ [46].

\section{Opium as an impartial factor}

A cross-sectional study on 304 patients with acute MI showed that patients with and without opium use disorder did not have any difference in the development of arrhythmia [47].

\section{Opium and ejection fraction (EF)}

\section{Opium as a potential risk factor for decreasing EF}

It was previously shown that patients with a history of opium use had a significantly lower EF compared to those who did not use opium, although the functional class was similar between the two groups [24]. A study of 232 diabetic CAD patients showed that EF was significantly lower in opium users with a statistically similar functional class [22]. In addition, two other studies conducted on patients undergoing CABG showed that EF was significantly lower in opium users [21, 48].

In 1993, 65 patients who used opium and ephedrone were investigated by 24-h Holter monitor, ECG, and echocardiography. Although none of the patients had heart failure and cardiac muscle contractions were normal, echocardiography showed abnormal changes in the left ventricle including ventricular enlargement, reduced $\mathrm{EF}$, and systolic shortening of myocardial fibers which were indicators of a decline in the compensatory mechanisms of the myocardium in substance users [42]. In 
another study conducted on patients undergoing coronary artery grafting surgery, 50 opium users and 50 non-users were compared regarding EF before the surgery. The results showed that average EF was lower in the opium users, but this difference was not statistically significant $(P=0.236)$. When the patients were categorized into the two subgroups of those with normal and abnormal EF, it was revealed that $56 \%$ of the patients with opium use disorder had abnormal EF (versus only 36\% abnormal EF in non-opium users) before the surgery showing a significant difference. Left ventricular end-diastolic pressure was also higher in addicted patients [49].

Garg reported a case of poisoning due to the consumption of raw opium resulting in severe depression in a 13year-old female who did not have any relevant medical history. On presentation, the patient was breathing shallowly, and the heart rate was over $150 \mathrm{bpm}$ accompanied by hypotension $(60 / 40 \mathrm{mmHg})$. Two-dimensional echocardiography showed left ventricular hypokinesia together with an LVEF of $10 \%$. Heart enzymes had also increased $(\mathrm{CK}-\mathrm{MB}=34.71 \mathrm{ng} / \mathrm{mL}$ [normal range; $1.39-6.22 \mathrm{ng} / \mathrm{mL}$ ] and troponin I: $0.32 \mathrm{ng} / \mathrm{mL}$ [normal range: $\leq 0.014 \mathrm{ng}$ / $\mathrm{mL}]$ ). The patient recovered eventually, and EF increased to $56 \%[50]$.

\section{Potential mechanism}

Opium contains 80 different alkaloids, such as morphine and codeine. Morphine is a $\mu$ receptor agonist responsible for cardiovascular complications, including histamine release, and results in bradycardia, vasodilation, hypotension, and decreased cardiac output [94]. It has also been demonstrated that increased contractile response due to the stimulation of rat cardiac betaadrenergic receptors is reversed through delta receptor agonism [95].

\section{Opium as an impartial factor}

A study on two groups of 228 diabetic patients undergoing angiography showed that EF was similar between opium users and non-users [12]. Roohafza et al. claimed that EF was similar between 126 opium users and nonusers with acute MI on presentation and within one year after that [26]. EF and Killip class were similar between addicted and non-addicted patients in studies on 460 and 304 MI patients [32, 47]. Another study evaluating 53 patients with CAD and 33 patients with normal angiography concluded that EF was similar between patients with and without opium use disorder [16].

The results of two other descriptive studies on 782 and 200 male patients undergoing CABG also showed that the functional class and EF before the surgery and six months after that were similar between case and control groups [3, 51]. Evaluations performed on 325 CABG candidates (117 opium users and 208 non-users) showed that there was no relationship between the duration of opium use and EF before and after surgery [1]. Davoodi et al. also confirmed the results mentioned above [38]. Sharafi et al. retrospectively investigated 1545 male patients undergoing PCI (including 350 opium users) using Tehran Heart Center data bank and showed that addicted and non-addicted patients were similar regarding the extent of the lesion and EF [33]. Similar results were withdrawn from other studies, all confirming that $\mathrm{EF}$, the emergence of heart failure after MI, and Killip class were similar between opium dependent and nondependent patients [23, 39, 52-54].

\section{Opium and mortality in patients with CAD Opium as a potential risk factor}

A cohort study of 566 CABG patients followed the patients for 6.5 years to obtain their survival rate, which was reported to be $86.6 \%$ in opium users and $92.7 \%$ in non-users (Hasard Ratio:2.16; CI 95\%: 0.96-4.84; P:0.06) [24]. The rate of mortality, angina after MI, cardiogenic shock, and cardiac arrest were shown to be significantly higher in opium users in another study [37], a result reported by other studies in this area, as well [39]. Najafi et al. showed that based on the EuroSCORE criteria, which is a method of predicting the possibility of mortality in patients undergoing heart surgery, the average score obtained from addicted patients was significantly higher than non-addicts [21]. In a cohort study conducted among 50,045 patients, opium use was suggested as a risk factor for mortality in these patients, even those who had used trivial amounts of opium. The use of opium significantly increased the overall risk of mortality $(\mathrm{HR}=1.86$; 95\% CI: $1.68-2.06)$ and the mortality due to different causes such as circulatory diseases $(\mathrm{HR}=1.81)$ and cancer $(\mathrm{HR}=1.61)$. Among the deaths due to blood circulation, opium use increased the risk of IHD (HR = 1.9; 95\% CI: 1.57-2.29) and cerebrovascular events [55].

\section{Opium as a potential protective factor}

A study of 460 MI patients revealed that inpatient mortality rate was lower in those with opium use disorder. Although this difference was not significant, in those with anterior wall infarction, the mortality was significantly higher in non-users [32].

\section{Opium as an impartial factor}

A retrospective study evaluating one-year major adverse cardiac events (e.g., cardiac death, non-fatal MI, need for target vessel revascularization, and target lesion revascularization) in 1545 patients revealed no significant difference between patients with and without opium use disorder $(P=0.312)$ [33]. Roohafza et al. claimed that the initial death rate was similar between these two groups. Also, they mentioned that one-year mortality and 
morbidity rates were also similar between them [26]. Another study demonstrated that although hospitalization period was more prolonged in opium users, hospital mortality, requiring re-hospitalization or re-vascularization, and the six-month mortality rate were still the same between them [38]. In 334 ST-elevation myocardial infarction (STEMI) patients, hospital outcome, mortality rate six months after MI, chest pain, and pulmonary edema were not different between the two groups [53].

Harati et al. evaluated the relationship between opium use, mortality rate and cardiac arrest in $400 \mathrm{MI}$ patients. They concluded there was no significant difference between addicted and non-addicted groups regarding the study outcomes, although opium users were more commonly hospitalized due to cardiac problems [56].

Short-term postoperative complications were evaluated in a study by Safaii et al., which reported that rates of complications after surgery, rehospitalization in the ICU, hospitalization period after the surgery, and hospital mortality were similar between the groups [3]. Other studies reported similar insignificant differences between the two groups of patients regarding hospital mortality rate, early postoperative complications, and duration of intubation and ICU admission [6, 29, 47, 52, 57, 58].

\section{Opium and response to treatment}

Jazi et al. examined the response to thrombolytic treatment in opium users in comparison to non-users and showed the beneficial effects of opium on the outcomes of thrombolytic therapy. Better response to thrombolytic agents indicates a smaller infarct size. Q-wave and STresolution after streptokinase injection were 63.8 and $47.2 \%$ in users and non-users, respectively $(P<0.05)$ [96]. The effect of opium use disorder on resistance to aspirin was also investigated in 260 patients suffering from angina, considering the measurement of 11dehydroxy thromboxane B2 level (UTXB2) in urine samples as the index for aspirin resistance. It was found that aspirin resistance was significantly higher in patients with opium use disorder [97].

\section{Discussion}

Most of the included studies did not support the protective effects of opium against CADs. Hasandokht [9] conducted a systematic review suggesting that opium did not have protective effects against CADs. It was also reported that age at the onset of CADs and hospital mortality were not protected by opium use. In another review article, it was noted that opium did not improve cardiovascular diseases. Instead, it harmed glycemic control, blood pressure, and lipid profile and caused atherosclerosis [40]. In addition to the adverse effect of opium on atherosclerosis of coronary arteries, some studies also noted that opium could be a risk factor for carotid stenosis [6]. The data from a large prospective cohort study showed the increased risk of death from circulatory diseases associated with opium consumption [55]. Some probable confounding factors should be considered in interpreting the results and design of the study. a) Traditionally, most opium users smoked tobacco. Hence, cigarette smoking should be considered as a confounding factor that was not reported in some included studies, which limits the generalizability of results. b) The dose of opium and the length, and frequency of opium use are also the issues that researchers should take into account. c) Since the opium available in the illicit market is not a pure substance and contains impurities such as lead, specifically in Iran, this factor can cause a difference in the results of the studies conducted on opium in different places around the world. To determine the exact effects of opium use on CADs further studies are required in this area.

\section{Conclusion}

Although the results are controversial, most of the studies mentioned above did not support the protective effects of opium against cardiovascular problems. Future studies are recommended to evaluate the effect of the route and amount of opium use on CADs and the interaction between opium and cigarette smoking. Unfortunately, misconceptions as to the positive effects of opium are widespread, and healthy people, as well as patients with heart diseases or diabetes, should be informed of the dangerous effects of opium use on cardiometabolic diseases. Also, is recommended to promote the knowledge of medical communities and staff on the potential health consequences of opium consumption. Future studies should aim to identify the appropriate strategies to treat substance use disorder for primary and secondary prevention of cardiovascular problems.

\footnotetext{
Abbreviations

CNS: Central nervous system; CVDs: Cardiovascular diseases; IHD: Ischemic heart disease; OR: Odds ratio; MESH: Medical Subject Headings; MCD: Microvascular coronary dysfunction; TG: Triglyceride; LDL: Low-density lipoprotein; CRP: C-reactive protein; LPA: Lysophosphatidic acid; ApoB: Apolipoprotein B; LDH: High-density lipoprotein; ALT: Alanine aminotransferase; AST: Aspartate aminotransferase; PAl-1: Plasminogen Activator Inhibitor-1; CHD: Congestive heart disease; CABG: Coronary artery bypass grafting; MI: Myocardial infarction; CKmb: Creatine kinase-MB; ECG: Electrocardiography; PCl: Percutaneous coronary intervention; ICU: Intensive care unit; STEMI: ST-elevated myocardial infarction; PVCs: Premature ventricular contractions; VT: Ventricular tachycardia; VF: Ventricular fibrillation; EF: Ejection fraction; LVEF: Left ventricular ejection fraction; HR: Hazard ratio; UTXB2: Urinary 11-dehydroxy thromboxane B2 level
} 


\section{Authors' contributions}

$\mathrm{SN}, \mathrm{SG}, \mathrm{KK}, \mathrm{NZ}, \mathrm{SA}, \mathrm{OM}$ contributed to the conception, design, and preparation of the manuscript. SN, SG, KK conducted the data collection and contributed to acquisition and interpretation. SN, NZ, OM made substantial contributions in drafting the manuscript and revising it critically for important intellectual content. All authors have read and approved the final version of the manuscript

\section{Funding}

No funding was obtained for this study.

\section{Availability of data and materials}

Not Applicable.

\section{Ethics approval and consent to participate}

Not applicable.

\section{Consent for publication}

Not Applicable.

\section{Competing interests}

Authors have no competing interests.

\section{Author details}

${ }^{1}$ Medical Toxicology and Drug Abuse Research Center (MTDRC), Birjand University of Medical Sciences, Birjand, Iran. ${ }^{2}$ Student Research Committee, Birjand University of Medical Sciences, Birjand, Iran. ${ }^{3}$ Department of Clinical Toxicology, Loghman-Hakim Hospital, Shahid Beheshti University of Medical Sciences, Tehran, Iran. ${ }^{4}$ Faculty of nursing and midwifery, Birjand University of Medical Sciences, Birjand, Iran. ${ }^{5}$ Rocky Mountain Poison and Drug Safety, Denver Health and Hospital Authority, 1391 Speer Blvd, 777 Bannock St. MC 0180, Denver, CO 80204, USA.

\section{Received: 15 January 2020 Accepted: 8 April 2020}

\section{Published online: 17 April 2020}

\section{References}

1. Aghadavoudi O, Eizadi-Mood N, Najarzadegan MR. Comparing cardiovascular factors in opium abusers and non-users candidate for coronary artery bypass graft surgery. Adv Biomed Res. 2015;4:12. https://doi. org/10.4103/2277-9175.148294. eCollection 2015.

2. Maghsoudi B, Khademi S, Akhlagh S, Khosravi M, Azemati S. Effect of opium addiction on perioperative needs to inotropic agents in coronary artery bypass surgery: a case-control study. Shiraz E Med J. 2012;13(1):5-12.

3. Safaii N, Kazemi B. Effect of opium use on short-term outcome in patients undergoing coronary artery bypass surgery. Gen Thorac Cardiovasc Surg. 2010;58(2):62-7.

4. Adelborg K, Horváth-Puhó E, Schmidt M, Munch T, Pedersen L, Nielsen PH et al. Thirty-year mortality after coronary artery bypass graft surgery: a Danish nationwide population-based cohort study. Circulation. 2017;10(5): e002708.

5. CAd S, MABd O, Brandi AC, PHH B, JdCM B, MAd S, et al. Risk factors for mortality of patients undergoing coronary artery bypass graft surgery. Braz Cardiovasc Surg. 2014;29(4):513-20.

6. Shirani S, Shakiba M, Soleymanzadeh M, Esfandbod M. Can opium abuse be a risk factor for carotid stenosis in patients who are candidates for coronary artery bypass grafting? Cardiol J. 2010;17(3):254-8.

7. Moezi SA, Azdaki N, Kazemi T, Moghaddam H, Partovi N, Hamidi F, et al. Effects of opium use on cardiovascular mortality: a critical appraisal of a topic. Iran J Public Health. 2019;48(10):1937.

8. Nakhaee S, Amirabadizadeh A, Qorbani M, Lamarine RJ, Mehrpour O. Opium use and cardiovascular diseases: a systematic review and meta-analysis. Crit Rev Toxicol. 2020:https://doi.org/https://doi.org/10.1080/10408444.2020. 1740972.

9. Hasandokht T, Salari A, Pour SS, Tirani HD, Shad B, Rajabi E. Does opium have benefit for coronary artery disease? A systematic review. Res Cardiovas Med. 2018:7(2):51.

10. Sadeghian S, Darvish S, Davoodi G, Salarifar M, Mahmoodian M, Fallah N, et al. The association of opium with coronary artery disease. Eur J Cardiovasc Prev Rehabil. 2007;14(5):715-7.
11. Sadeghian S, Graili P, Salarifar M, Karimi AA, Darvish S, Abbasi SH. Opium consumption in men and diabetes mellitus in women are the most important risk factors of premature coronary artery disease in Iran. Int J Cardiol. 2010;141(1):116-8.

12. Hosseini SK, Masoudkabir F, Vasheghani-Farahani A, Alipour-Parsa S, Fathollahi MS, Rahimi-Foroushani A, et al. Opium consumption and coronary atherosclerosis in diabetic patients: a propensity score-matched study. Planta Med. 2011;77(17):1870-5.

13. Darabad BR, Vatandust J, Khoshknab MP, Poorrafsanjani MH. Survey of the effect of opioid abuse on the extent of coronary artery diseases. Global J Health Sci. 2014;6(7):83.

14. Kazerani H, Rai A, Heidary-Moghadam R, Asgari N, Nowroozi M, Rasouli MH. Relationship between using raw opium and opioids with coronary artery stenosis based on coronary an-giography findings. J Biol Todays World. 2014;3:71-6.

15. Nadimi AE, Amiri FP, Fathollahi MS, Hassanshahi G, Ahmadi Z, Sayadi AR. Opium addiction as an independent risk factor for coronary microvascular dysfunction: a case-control study of 250 consecutive patients with slowflow angina. Int J Cardiol. 2016:219:301-7.

16. Masoomi M, Ramezani MA, Karimzadeh H. The relationship of opium addiction with coronary artery disease. Int J Prev Med. 2010;1 (3):182.

17. Mohammadi A, Darabi M, Nasry M, Saabet-Jahromi M-J, Malek-Pour-Afshar R, Sheibani H. Effect of opium addiction on lipid profile and atherosclerosis formation in hypercholesterolemic rabbits. Exp Toxicol Pathol. 2009;61(2): 145-9.

18. Dressler FA, Roberts WC. Modes of death and types of cardiac diseases in opiate addicts: analysis of 168 necropsy cases. Am J Cardiol. 1989;64(14): 909-20.

19. Marmor M, Penn A, Widmer K, Levin Rl, Maslansky R. Coronary artery disease and opioid use. Am J Cardiol. 2004;93(10):1295-7.

20. Massomi M, Shahesmaeili A, Mirzazadeh A, Tavakoli M, Ali AZ. Opium addiction and severity of coronary artery disease: a case-control study. J Res Med Sci. 2009;15(1):27-32.

21. Najafi M, Sheikhvatan M. Does analgesic effect of opium hamper the adverse effects of severe coronary artery disease on quality of life in addicted patients? Anesthesiol Pain Med. 2012;2(1):22.

22. Najafi M, Sheikhvatan M. Plausible impact of dietary habits on reduced blood sugar in diabetic opium addicts with coronary artery disease. Int Cardiovas Res J. 2012;6(3):75

23. Rezvani MR, Ghandehari K. Is opium addiction a risk factor for ischemic heart disease and ischemic stroke? J Res Med Sci. 2012;17(10):958.

24. Najafi M, Jahangiry L, Mortazavi SH, Jalali A, Karimi A, Bozorgi A. Outcomes and long-term survival of coronary artery surgery: the controversial role of opium as risk marker. World J Cardiol. 2016;8(11):676.

25. Shahryari J, Poormorteza M, Noori-Sorkhani A, Divsalar K, Abbasi-Oshaghi E. The effect of concomitant ethanol and opium consumption on lipid profiles and atherosclerosis in golden Syrian Hamster's aorta. Addict Health. 2013; 5(3-4):83.

26. Roohafza H, Sadeghi M, Haghani P, Shokouh P, Sarrafzadegan N. Opium decreases the age at myocardial infarction and sudden cardiac death: a long-and short-term outcome evaluation. Arch Iran Med. 2013;16(3):154

27. Niaki MRK, Hamid M, Farshidi F, Mohammadpour M, Omran MTS. Evaluation of the role of opium addiction in acute myocardial infarction as a risk factor. Caspian J Intern Med. 2013;4(1):585.

28. Li L, Setoguchi S, Cabral H, Jick S. Opioid use for noncancer pain and risk of myocardial infarction amongst adults. J Intern Med. 2013;273(5):511-26.

29. Bartolucci J, Nazzal C, Verdugo FJ, Prieto J, Sepulveda P, Corbalan R. Characteristics, management, and outcomes of illicit drug consumers with acute myocardial infarction. Revista medica de Chile. 2016;144(1):39-46.

30. Maleki A, Nabatchi B. The Opioid Effects On The Cardiac Markers And Left Ventricular Contractility In Acute Myocardial Infarction Patients. Internet J Cardiol. 2012;10(2):1-4.

31. Joukar S, Najafipour H, Malekpour-Afshar R, Mirzaeipour F, Nasri HR. The effect of passive opium smoking on cardiovascular indices of rabbits with normal and ischemic hearts. Open Cardiovas Med J. 2010;4:1.

32. Dehghani F, Masoomi M, Haghdoost AA. Relation of opium addiction with the severity and extension of myocardial infarction and its related mortality. Addict Health. 2013;5(1-2):35.

33. Sharafi A, Hosseini HRP, Jalali A, Salarifar M, Nematipour E, Shojanasab M, et al. Opium consumption and mid-term outcome of percutaneous coronary intervention in men. J Tehran Univ Heart Center. 2014;9(3):115. 
34. Okubo S, Tanabe Y, Takeda K, Kitayama M, Kanemitsu S, Kukreja RC, et al. Ischemic preconditioning and morphine attenuate myocardial apoptosis and infarction after ischemia-reperfusion in rabbits: role of $\delta$-opioid receptor. Am J Phys Heart Circ Phys. 2004;287(4):H1786-H91.

35. Aitchison KA, Baxter GF, Awan MM, Smith RM, Yellon DM, Opie LH. Opposing effects on infarction of delta and kappa opioid receptor activation in the isolated rat heart: implications for ischemic preconditioning. Basic Res Cardiol. 2000;95(1):1-10.

36. Azimzade SB, Gholamreza Y, Narooey S. A case-control study of effect of opium addiction on myocardial infarction. Am J Appl Sci. 2005:2:1134-5.

37. Najar L, Vakili B, Behnam VH. A comparative study of early complications of acute myocardial infarction in addicted and non-addicted patients at ccu of heshmatyya hospital Sabzevar, Iran. J Sabzevar Univ Med Sci. 2001;8(20):96-103.

38. Davoodi G, Sadeghian S, Akhondzadeh S, Darvish S, Alidoosti M, Amirzadegan A. Comparison of specifications, short term outcome and prognosis of acute myocardial infarction in opium dependent patients and nondependents. J Tehran Univ Heart Center. 2006;1(1):48-53.

39. Rostamzadeh A, Khademvatani K. Comparison of myocardial infarction outcome in opium dependent and non-dependent patients. J Urmia Univ Med Sci. 2016;27(3):208-14.

40. Masoudkabir F, Sarrafzadegan N, Eisenberg MJ. Effects of opium consumption on cardiometabolic diseases. Nat Rev Cardiol. 2013;10(12):733.

41. Mirzaiepour F, Dadras M, Forood A. The Effect of Opium Addiction on Cardiac Arrhythmia after Acute Myocardial Infarction. J Kerman Univ Med Sci. 2012;19(4):367-74

42. Gorgaslidze A, Săfullaeva M, Kuz'mina M, Golitsina L, Smetnev A. Cardiac arrhythmia and myocardial contraction in opium and ephedrone addiction. Kardiologiia. 1993;33(1):14-6.

43. Najafipour $\mathrm{H}$, Joukar $\mathrm{S}$. Combination of opium smoking and hypercholesterolemia augments susceptibility for lethal cardiac arrhythmia and atherogenesis in rabbit. Environ Toxicol Pharmacol. 2012;34(2):154-9.

44. Coles JA Jr, Sigg DC, laizzo PA. Role of k-opioid receptor activation in pharmacological preconditioning of swine. Am J Phys Heart Circ Phys. 2003; 284(6):H2091-H9.

45. Gad A, Morelli P, Decristofaro J. Perinatal isolated atrial flutter associated with maternal cocaine and opiate use in a late preterm infant. J Matern Fetal Neonatal Med. 2010;23(9):1062-5.

46. Sabzi F, Zokaei AH, Moloudi AR. Predictors of atrial fibrillation following coronary artery bypass grafting. Clin Med Insights. 2011;5:CMC. S7170.

47. Javadi HR, Allami A, Mohammadi N, Alauddin R. Opium dependency and inhospital outcome of acute myocardial infarction. Med J Islam Repub Iran 2014;28:122. PubMed PMID: 25679001. Pubmed Central PMCID: Pmc4313445. Epub 2015/02/14. eng.

48. Eizadi-Mood N, Aghadavoudi O, Najarzadegan MR, Fard MM. Prevalence of delirium in opium users after coronary artery bypass graft surgery. Int J Prev Med 2014 Jul;5(7):900-906. PubMed PMID: 25105003. Pubmed Central PMCID: Pmc4124569. Epub 2014/08/12. eng.

49. Kiani A, Aghdaee N, Azarfarin R, Farasarkish R, Naddafnia H. Evaluation of prolong effect of opium on cardiac function in coronary artery bypass graft. J Iran Soc Anaesthesiol Intensive Care. 2007;29(60):60-6.

50. Garg P, Hitawala AA, Agarwal M. Cardiotoxic effects of raw opium. Indian J Crit Care Med. 2018;22(1):46.

51. Safaei N. Outcomes of coronary artery bypass grafting in patients with a history of opiate use. Pak J Biol Sci. 2008;11(22):2594.

52. Azarasa M, Azarfarin R, Changizi A, Alizadehasl A. Substance use among Iranian cardiac surgery patients and its effects on short-term outcome. Anesth Analg. 2009;109(5):1553-9.

53. Mousavi M, Kalhor S, Tahmasebi J, Alizadeh M. opium Addiction And Early And 6-month Outcomes Of Patients With St Elevation Myocardial Infarction: 173. Cardiology. 2015;131:185.

54. Hadadzadeh M, Abdollahi M, Forozannia K, Seyfaddini A. Investigating Effect of Drug Use on Short-term Complications and Bleeding in Patients Undergoing Off-pump CABG (OPCAB). SSU_J. 2013;21(3):26570 .

55. Khademi H, Malekzadeh R, Pourshams A, Jafari E, Salahi R, Semnani S, et al. Opium use and mortality in Golestan cohort study: prospective cohort study of 50000 adults in Iran. Bmj. 2012;344:e2502.

56. Harati H, Shamsi A, Moghadam MF, Zadeh FSS, Ghazi A. The Mortality Rate of Myocardial Infraction Patients With and Without Opium Dependen. Int J High Risk Behav Addict. 2015;4(3):e22576.
57. Bafghi SS, Rafiei M, Bahadorzadeh L, Namayeh S, Soltani M, Andishmand MMA. Is opium addiction a risk factor for acute myocardial infarction? Acta medica Iranica. 2005;43(3):218-22.

58. Amouzeshi A, Dolatabadi M, Nakhaee S, Maleki MH, Mehrpour O. Comparing short-term mortality in opium users and non-users candidate for coronary artery bypass graft surgery. 2017;5(1 and 2):13-17.

59. Saadat H, Ziai SA, Ghanemnia M, Namazi MH, Safi M, Vakili H, et al. Opium addiction increases interleukin 1 receptor antagonist (IL-1Ra) in the coronary artery disease patients. PLoS One. 2012;7(9):e44939.

60. Naderi G, Asgary S, Sadeghi M, Sabetnezhad Z, Tansaz M. Comparing plasma level of CRP, factor VII, fibrinogen; platelet counts, systolic and diastolic blood pressure in smokers with opium addicted smokers. J Qazvin Univ of Med Sci. 2005:9(2):3-7.

61. Asgary S, Sarrafzadegan N, Naderi G-A, Rozbehani R. Effect of opium addiction on new and traditional cardiovascular risk factors: do duration of addiction and route of administration matter? Lipids Health Dis. 2008;7(1):42.

62. Najafipour $\mathrm{H}$, Beik $\mathrm{A}$. The impact of opium consumption on blood glucose, serum lipids and blood pressure, and related mechanisms. Front Physiol. 2016;7:436.

63. Karam GA, Reisi M, Kaseb AA, Khaksari M, Mohammadi A, Mahmoodi M. Effects of opium addiction on some serum factors in addicts with noninsulin-dependent diabetes mellitus. Addict Biol. 2004;9(1):53-8.

64. Kouros D, Tahereh H, Mohammadreza A, Minoo MZ. Opium and heroin alter biochemical parameters of human's serum. Am J Drug Alcohol Abuse. 2010; 36(3):135-9.

65. Najafipour H, Joukar S, Malekpour-Afshar R, Mirzaeipour F, Nasri HR. Passive opium smoking does not have beneficial effect on plasma lipids and cardiovascular indices in hypercholesterolemic rabbits with ischemic and non-ischemic hearts. J Ethnopharmacol. 2010;127(2):257-63.

66. Masoomi M, Azdaki N, Shahouzehi B. Elevated plasma Homocysteine concentration in opium-addicted individuals. Addict Health. 2015;7(34):149.

67. Moloudi A, Sabzi F, Shahhosaini E. Analysis of coagulation tests, proteins C and $\mathrm{S}$, and plasma fibrinogen in addicts and non-addicts with coronary artery disease. J Kermanshah Univ Med Sci. 2014;17(12):812-4.

68. Azdaki N, Zardast M, Anani-Sarab G, Abdorrazaghnaejad H, Ghasemian MR, Saburi A. Comparison between Homocysteine, fibrinogen, PT, PTT, INR and CRP in male smokers with/without addiction to opium. Addict Health. 2017; 9(1):17.

69. Masoomi M, Nasri H, Farajpour F. Comparison of plasma fibrinogen level in opium addict men with non-addict men; 2002.

70. Forood A, Malekpour-Afshar R, Mahdavi A. Serum level of plasminogen activator inhibitor type-1 in addicted patients with coronary artery disease. Addict Health. 2014;6(3-4):119.

71. Shahouzehi B, Shokoohi M, Najafipour $H$. The effect of opium addiction on serum adiponectin and leptin levels in male subjects: a case control study from Kerman coronary artery disease risk factors study (KERCADRS). Excli J. 2013;12:916

72. Hayatbakhsh MM, Oghabian Z, Conlon E, Nakhaee S, Amirabadizadeh AR, Zahedi MJ, et al. Lead poisoning among opium users in Iran: an emerging health hazard. Subst Abuse Treat Prev Policy. 2017;12(1):43.

73. Alinejad S, Aaseth J, Abdollahi M, Hassanian-Moghaddam H, Mehrpour O. Clinical aspects of opium adulterated with lead in Iran: a review. Basic Clin Pharmacol Toxicol. 2018;122(1):56-64

74. Nakhaee S, Mehrpour O. Opium addiction as new source of lead poisoning: an emerging epidemic in Iran. Excli J. 2018;17:513.

75. Navas-Acien A, Guallar E, Silbergeld EK, Rothenberg SJ. Lead exposure and cardiovascular disease_-a systematic review. Environ Health Perspect. 2006; 115(3):472-82.

76. Najafipour H, Masoomi M, Shahesmaeili A, Haghdoost AA, Afshari M, Nasri $H R$, et al. Effects of opium consumption on coronary artery disease risk factors and oral health: results of Kerman coronary artery disease risk factors study a population-based survey on 5900 subjects aged 15-75 years. Int J Prev Med. 2015;6(1):42. https://doi.org/10.4103/2008-7802.157470.

77. Ghazavi A, Mosayebi G, Solhi H, Rafiei M, Moazzeni SM. Serum markers of inflammation and oxidative stress in chronic opium (Taryak) smokers. Immunol Lett. 2013;153(1-2):22-6.

78. Gülçın I, Beydemır S, Alici HA, Elmastas M, Büyükokuroğlu ME. In vitro antioxidant properties of morphine. Pharmacol Res. 2004;49(1):59-66.

79. Gettler JF. Hypocholesterolemia in substance abusers. South Med J. 1991; 84(7):937. 
80. Schultz JEJ, Hsu AK, Gross GJ. Ischemic preconditioning in the intact rat heart is mediated by $\delta 1$-but not $\mu$-or k-opioid receptors. Circulation. 1998; 97(13):1282-9.

81. Bolling SF, Badhwar V, Schwartz CF, Oeltgen PR, Kilgore K, Su T-P. Opioids confer myocardial tolerance to ischemia: interaction of delta opioid agonists and antagonists. J Thorac Cardiovasc Surg. 2001;122(3):476-81.

82. Sigg DC, Coles JA Jr, Oeltgen PR, laizzo PA. Role of $\delta$-opioid receptor agonists on infarct size reduction in swine. Am J Phys Heart Circ Phys. 2002; 282(6):H1953-H60.

83. Schultz JEJ, Hsu AK, Nagase H, Gross GJ. TAN-67, a 81-opioid receptor agonist, reduces infarct size via activation of Gi/o proteins and KATP channels. Am J Phys Heart Circ Phys. 1998;274(3):H909-H14.

84. Schultz JJ, Hsu AK, Gross GJ. Ischemic preconditioning and morphineinduced cardioprotection involve the delta ( $\delta$ )-opioid receptor in the intact rat heart. J Mol Cell Cardiol. 1997:29(8):2187-95.

85. Schultz JEJ, Gross GJ. Opioids and cardioprotection. Pharmacol Ther. 2001; 89(2):123-37.

86. Soroosh D, Neamatshahi M, Zarmehri B, Nakhaee S, Mehrpour O. Druginduced prolonged corrected QT interval in patients with methadone and opium overdose. Subst Abuse Treat Prev Policy. 2019;14(1):8.

87. Mahmoodi Z, Keikhaie KR, Salarzaei M, Havasian MR. The incidence of different kinds of cardiac arrhythmia after myocardial infarction in smokers and opium abusers hospitalized in imam Ali Hospital, Zahedan, 2016. J Pharm Sci Res. 2017:9(7):1243.

88. Mirzaiepour F, Dadras M, Forood A, Najafipour H, Shokoohi M. The effect of opium addiction on arrhythmia following acute myocardial infarction. Acta Medica Iranica. 2012;50(10):670-675. PubMed PMID: 23275281. Epub 2013/ 01/01. eng.

89. Hill-Pryor C, Lindsey D, Lapanowski K, Dunbar JC. The cardiovascular responses to mu opioid agonist and antagonist in conscious normal and obese rats. Peptides. 2006 Jun;27(6):1520-1526. PubMed PMID: 16293344. Epub 2005/11/19. eng.

90. Feuerstein $\mathrm{G}$, Siren $\mathrm{A}-1$. the opioid peptides. A role in hypertension? Hypertension. 1987;9(6):561-5.

91. Paakkari P, Paakkari I, Feuerstein G, Siren AL. Evidence for differential opioid mu 1- and mu 2-receptor-mediated regulation of heart rate in the conscious rat. Neuropharmacology. 1992 Aug;31 (8):777-782. PubMed PMID: 1356253. Epub 1992/08/01. eng.

92. Pugsley MK, Saint DA, Penz MP, Walker MJ. Electrophysiological and antiarrhythmic actions of the kappa agonist PD 129290, and its R,R (+)-enantiomer, PD 129289. Br J Pharmacol 1993;110(4):1579-1585. PubMed PMID: 8306104. Pubmed Central PMCID: Pmc2175892. Epub 1993/12/01. eng.

93. Malinowska B, Piszcz J, Koneczny B, Hryniewicz A, Schlicker E. Modulation of the cardiac autonomic transmission of pithed rats by presynaptic opioid OP4 and cannabinoid CB1 receptors. Naunyn Schmiedeberg's Arch Pharmacol 2001 Sep;364(3):233-241. PubMed PMID: 11521166. Epub 2001/ 08/25. eng.

94. Behzadi M, Joukar S, Beik A. Opioids and cardiac arrhythmia: a literature review. Med Princ Pract. 2018:27(5):401-14.

95. Pepe S, Altschuld R, Lakatta E. 8-Opioid peptide receptors activate pertussis toxin-sensitive and pertussis toxin-insensitive pathways during myocardial contraction. J Mol Cell Cardiol. 2001;33(6):A92.

96. Jazi SMH, Nazary IA, Behjati M. Response to thrombolytic agents in acute myocardial infarction in opium abusers versus non-abusers. J Res Pharm Pract. 2012;1(1):34.

97. Forood A, Malekpour-Afshar R, Sarnevesht J. Effect of opium addiction on aspirin resistance in stable angina pectoris. Addict Health. 2014;6(1-2):7.

\section{Publisher's Note}

Springer Nature remains neutral with regard to jurisdictional claims in published maps and institutional affiliations.

Ready to submit your research? Choose BMC and benefit from:

- fast, convenient online submission

- thorough peer review by experienced researchers in your field

- rapid publication on acceptance

- support for research data, including large and complex data types

- gold Open Access which fosters wider collaboration and increased citations

- maximum visibility for your research: over $100 \mathrm{M}$ website views per year

At $\mathrm{BMC}$, research is always in progress.

Learn more biomedcentral.com/submissions 